\title{
DETERMINANT EXPRESSION \\ OF SELBERG ZETA FUNCTIONS. I
}

\author{
SHIN-YA KOYAMA
}

\begin{abstract}
We show that for $\operatorname{PSL}(2, \mathbf{R})$ and its congruence subgroup, the Selberg zeta function with its gamma factors is expressed as the determinant of the Laplacian. All the gamma factors are calculated explicitly. We also give an explicit computation to the contribution of the continuous spectrum to the determinant of the Laplacian.
\end{abstract}

\section{INTRODUCTION}

Throughout this paper we fix $G$ to be $\operatorname{PSL}(2, \mathbf{R})$ and $\Gamma$ its congruence subgroup. Selberg defined the following zeta function in [7]:

$$
Z(s):=\prod_{p} \prod_{n=0}^{\infty}\left(1-N(P)^{-s-n}\right),
$$

where $P$ runs through all the representatives of primitive hyperbolic conjugacy classes of $\Gamma$, and $N(P):=\alpha^{2}$ if the eigenvalues of $P$ are $\alpha$ and $\alpha^{-1}(|\alpha|>1)$. Selberg proves that $Z(s)$ has a meromorphic continuation to the whole $s$-plane and that $s=1$ is a simple zero. In this paper we complete $Z(s)$ to

$$
\widehat{Z}(s):=Z(s) Z_{I}(s) Z_{E}(s) Z_{P}(s)
$$

by supplying three factors. The main result in this paper says that $\hat{Z}(s)$ has a determinant expression as

$$
\widehat{Z}(s)=e^{c+c^{\prime} s(s-1)} \operatorname{det}(\Delta, s),
$$

where $\Delta$ is the Laplacian for the upper half-plane. Consequently, the coefficient of the first term of the Taylor expansion of $Z(s)$ around $s=1$ is expressed as

$$
Z^{\prime}(1)=\left(\operatorname{det}_{D}^{\prime} \Delta\right) \times(\text { elementary factor }),
$$

where $\operatorname{det}_{D}^{\prime}$ is the determinant composed of nonzero discrete spectrum. The symmetric functional equation is obvious because the discrete part of the determinant is invariant under $s \rightarrow 1-s$. When $\Gamma$ is torsion free and the quotient space $G / \Gamma$ is compact, this determinant expression is obtained by Voros [11], and Sarnak [6]. Moreover, when $\Gamma$ is $\operatorname{PSL}(2, \mathbf{Z})$, it was proved by $\operatorname{Kurokawa}$

Received by the editors February 21, 1989.

1980 Mathematics Subject Classification (1985 Revision). Primary 11F72. 
[5]. The outline of this paper is as follows. In $\S 2$, we recall the Selberg trace formula for $\operatorname{PSL}(2, \mathbf{R})$, which plays an important role in the proof of the main result. In $\S 3$, we give the analytic continuation of the spectral zeta function. It has not been done for a congruence subgroup $\Gamma$ so far. In $\S 4$, we define the determinant of Laplacian composed of both the discrete and the continuous spectrum. The discrete part is available from the spectral zeta function, while the continuous part derives from the corresponding terms of the Selberg trace formula. It turns out that in the continuous part of the determinant of the Laplacian, arithmetic zeta functions such as the Riemann zeta or Dirichlet $L$ functions arise. In $\S 5$ we have the determinant expression and in $\S 6$ we decide the constants $c$ and $c^{\prime}$ in (1.2).

In part II, S. Koyama, Determinant expression of Selberg zeta functions. II, Trans. Amer. Math. Soc. (to appear), we will have the determinant expression of Selberg zeta functions for $G=\operatorname{PSL}(2, \mathrm{C})$ and $\Gamma=\operatorname{PSL}(2, O)$, where $O$ is the integer ring of an imaginary quadratic field.

The author would like to express his profound gratitude to Professor N. Kurokawa. He introduced the author to the subject and has made a lot of valuable suggestions and encouragement in the whole process of producing this paper. Especially, the analytic continuation in $\S 3$ would not have been accomplished without his advice.

\section{The Selberg trace formula}

Let $\mathbf{H}$ be the upper half-plane,

$$
\mathbf{H}:=\{z=x+i y \in \mathbf{C} \mid y>0\},
$$

with the Riemannian metric $d s^{2}=\left(d x^{2}+d y^{2}\right) / y^{2}$. The group $G=\operatorname{PSL}(2, \mathbf{R})$ acts on $\mathbf{H}$ transitively by the usual linear fractional transformation. The fundamental domain of $\Gamma$ is denoted by $\Gamma \backslash \mathbf{H}$. The Laplacian is

$$
\Delta:=-y^{2}\left(\frac{\partial^{2}}{\partial x^{2}}+\frac{\partial^{2}}{\partial y^{2}}\right)
$$

which has the unique selfadjoint extension on $L^{2}(\Gamma \backslash \mathbf{H})$. We denote it by the same notation $\Delta$. The Selberg trace formula is the equality between two different expressions of the trace of an integral operator whose continuous spectrum is removed:

Theorem 2.1 (Venkov [9, Theorem 4.3.6, 4.4.4]). Let $h\left(r^{2}+\frac{1}{4}\right)$ be a function of a complex variable $r$ which satisfies the following assertions:

(1) As a function of $r, h\left(r^{2}+\frac{1}{4}\right)$ is holomorphic in the strip $\{r \in \mathbf{C} ;|\operatorname{Im}(r)|$ $\left.<\frac{1}{2}+\varepsilon\right\}$ for some $\varepsilon>0$.

(2) In that strip, $h\left(r^{2}+\frac{1}{4}\right)=O\left(\left(1+\left|r^{2}\right|\right)^{-1-\varepsilon}\right)$ and all of the series and integrals appearing below converge absolutely. 
Then the following identity holds:

$$
\begin{aligned}
\sum_{n=0}^{\infty} h\left(\lambda_{n}\right) & \\
& -\frac{1}{4 \pi} \int_{-\infty}^{\infty} \frac{\varphi^{\prime}}{\varphi}\left(\frac{1}{2}+i r\right) h\left(r^{2}+\frac{1}{4}\right) d r \\
& -\frac{K_{0}}{4} h\left(\frac{1}{4}\right) \\
= & \frac{\operatorname{vol}(\Gamma \backslash \mathbf{H})}{4 \pi} \int_{-\infty}^{\infty} r \tanh \left(r^{2}+\frac{1}{4}\right) d r \\
& +\sum_{P} \sum_{m=1}^{\infty} \frac{\log N(P)}{N(P)^{m / 2}-N(P)^{-m / 2}} g(m \log N(P)) \\
& +\frac{1}{2} \sum_{R}^{\nu} \sum_{m=1}^{\nu-1} \frac{1}{\nu \sin \pi m / \nu} \int_{-\infty}^{\infty} \frac{\exp (-2 \pi r m / \nu)}{1+\exp (-2 \pi r)} h\left(r^{2}+\frac{1}{4}\right) d r \\
& -K g(0) \log 2 \\
& +\frac{K}{4} h\left(\frac{1}{4}\right) \\
& -\frac{K}{2 \pi} \int_{-\infty}^{\infty} \psi \psi
\end{aligned}
$$

Notation is as follows: The set of the discrete spectrum of $\Delta$ is denoted by $\left\{\lambda_{n} \mid 0=\lambda_{0}<\lambda_{1} \leq \lambda_{2} \leq \cdots\right\}$. The volume of the fundamental domain is denoted by $\operatorname{vol}(\Gamma \backslash \mathbf{H})$. The function $g$ is the one that appears in the process of the Selberg transformation:

$$
g(u):=\frac{1}{2 \pi} \int_{-\infty}^{\infty} e^{-i r u} h\left(r^{2}+\frac{1}{4}\right) d r .
$$

As for sums in (2.1.H) (resp. (2.1.E)), $P$ (resp. $R$ ) runs through all the representatives of primitive hyperbolic (resp. elliptic) conjugacy classes of $\Gamma$. The number of cusps is denoted by $K$, and $\varphi$ is the determinant of the $(K \times K)$ matrix whose components are the functions which appear in the constant term of the Eisenstein series attached to $\Gamma$. The matrix is called the scattering matrix, and $\varphi$ is called the scattering determinant of $\Gamma$. If $\Phi(s)$ denotes the scattering matrix of $\Gamma, K_{0}$ is defined as

$$
K_{0}:=\lim _{s \rightarrow 1 / 2} \operatorname{tr}(\Phi(s))
$$

and $\psi$ is the di-gamma function $\Gamma^{\prime} / \Gamma$.

The method we use to name each term is the following: $\mathrm{D}$ and $\mathrm{C}$ correspond to the discrete and the removed continuous spectrum, and $\mathrm{I}, \mathrm{H}, \mathrm{E}$, and $\mathrm{P}$ are the contribution of the identity, hyperbolic, elliptic, and parabolic conjugacy classes, respectively. 
The following subgroups of $\operatorname{SL}(2, \mathbf{Z})$ is defined for a positive integer $N$ :

$$
\begin{aligned}
& \Gamma_{0}(N):=\left\{\left(\begin{array}{ll}
a & b \\
c & d
\end{array}\right) \in S L(2, \mathbf{Z}) ; c \equiv 0(\bmod N)\right\}, \\
& \Gamma_{1}(N):=\left\{\left(\begin{array}{ll}
a & b \\
c & d
\end{array}\right) \in \Gamma_{0}(N) ; a \equiv d \equiv 1(\bmod N)\right\}, \\
& \Gamma_{2}(N):=\left\{\left(\begin{array}{ll}
a & b \\
c & d
\end{array}\right) \in \Gamma_{1}(N) ; b \equiv 0(\bmod N)\right\} .
\end{aligned}
$$

The image of $\Gamma_{i}(N)(i=0,1,2)$ by the natural quotient map $\operatorname{SL}(2, \mathbf{Z}) \rightarrow$ $\operatorname{PSL}(2, \mathbf{Z})$ is also denoted by the same notation, for we will treat only subgroups of $\operatorname{PSL}(2, \mathbf{Z})$ in what follows in this paper. When there exists an integer $N$ such that

$$
\operatorname{PSL}(2, \mathbf{Z}) \supset \Gamma \supset \Gamma_{2}(N),
$$

we call $\Gamma$ a congruence subgroup of level $N$. For $\Gamma=\Gamma_{i}(N)(i=0,1,2)$, the scattering determinant is obtained by Huxley.

Theorem 2.3 (Huxley [3]). The scattering determinant of $\Gamma_{i}(N)(i=0,1,2)$ is

$$
\varphi(s)=(-1)^{\left(K-K_{0}\right) / 2}\left(\frac{\Gamma(1-s)}{\Gamma(s)}\right)^{K}\left(\frac{A}{\pi^{K}}\right)^{1-2 s} \prod_{\chi} \frac{L(2-2 s, \bar{\chi})}{L(2 s, \chi)} .
$$

Notation is as follows: $K$ and $K_{0}$ are as in Theorem 2.1. $L(s, \chi)$ is the Dirichlet $L$-function. Dirichlet characters which appear in the above product are what can be written as

$$
\chi(n)=\chi_{1}(n) \chi_{2}(n) \omega_{m_{1} m_{2}}(n) \quad(n \in \mathbf{Z}, n>0),
$$

where $\chi_{i}$ is a primitive Dirichlet character $\bmod q_{i}(i=1,2)$, and $\omega_{m_{1} m_{2}}$ is the trivial character $\bmod m_{1} m_{2}$. As for the above product over $\chi$, variables $q_{1}, q_{2}, m_{1}$, and $m_{2}$ run through all the positive integers satisfying the conditions below, and $\chi_{i}(i=1,2)$ runs through all possible Dirichlet characters.

Conditions. (a) $\Gamma_{2}(N) \cdots\left(m_{1}, m_{2}\right)=1, m_{1} q_{1}\left|N, m_{2} q_{2}\right| N$.

(b) $\Gamma_{1}(N) \cdots(a)$ and $m_{1}=1, q_{1} \mid m_{2}$.

(c) $\Gamma_{0}(N) \cdots(b)$ and $q_{1}=q_{2}, \chi_{1}=\chi_{2}$.

The products has $K$ terms and $A$ is a positive integer composed of the primes dividing $N$ :

$$
A= \begin{cases}\prod_{(\mathbf{a})} m_{1} m_{2} q_{1} N & \text { for } \Gamma_{2}(N), \\ \prod_{(\mathbf{b})} q_{1} N & \text { for } \Gamma_{1}(N), \\ \prod_{(\mathbf{c})} q_{1} N /\left(m_{2}, N / m_{2}\right) & \text { for } \Gamma_{0}(N) .\end{cases}
$$

By substituting the logarithmic derivative of (2.3) into (2.1.C1), Huxley rewrites the terms of continuous spectrum in $[3, \S 5]$ as follows. 
Theorem 2.3. When $\Gamma$ is $\Gamma_{i}(N)(i=0,1,2)$, the sum of the terms of (2.1.C1) and (2.1.C2) becomes

$$
\begin{aligned}
g(0) & \log \frac{A}{\pi^{K}} \\
& -2 \sum_{\chi} \sum_{n=1}^{\infty} \frac{\Lambda(n) \chi(n)}{n} g(2 \log n) \\
& +\frac{K}{2 \pi} \int_{-\infty}^{\infty}\left(r^{2}+\frac{1}{4}\right) \Psi\left(\frac{1}{2}+i r\right) d r \\
& -\frac{K_{0}}{4} h\left(\frac{1}{4}\right)
\end{aligned}
$$

where $\Lambda(n)$ is the von Mangoldt function, equal to $\log p$ if $n$ is a prime power, and zero otherwise.

\section{ANALYTIC CONTINUATION OF THE SPECTRAL ZETA FUNCTION}

The spectral zeta function is defined by

$$
\zeta(w, \Delta):=\sum_{n=1}^{\infty} \frac{1}{\lambda_{n}^{w}} .
$$

We will treat the function generalized by $s$,

$$
\zeta(w, s, \Delta):=\sum_{n=0}^{\infty} \frac{1}{\left(\lambda_{n}+s(s-1)\right)^{w}},
$$

for the purpose of associating the complete Selberg zeta function in $\S 5$. As the test function in the Selberg formula, we adopt the following function:

$$
h\left(r^{2}+\frac{1}{4}\right):=\exp \left(-t\left(r^{2}+\frac{1}{4}+s(s-1)\right)\right) \quad(t>0, s>1),
$$

which satisfies the assumptions of the trace formula. The correspnding $g(u)$ is

$$
g(u)=\frac{1}{\sqrt{4 \pi t}} \exp \left(-t\left(s-\frac{1}{2}\right)^{2}-\frac{u^{2}}{4 t}\right)
$$

Then the spectral zeta function $\zeta(w, s, \Delta)$ appears in the Mellin transformation of (2.1.D). Indeed,

$$
\begin{aligned}
\int_{0}^{\infty} & \sum_{n=0}^{\infty} \exp \left(-t\left(\lambda_{n}+s(s-1)\right)\right) t^{w} \frac{d t}{t} \\
& =\sum_{n=0}^{\infty} \int_{0}^{\infty} \exp \left(-t\left(\lambda_{n}+s(s-1)\right)\right)\left(t\left(\lambda_{n}+s(s-1)\right)\right)^{2} \frac{d t}{t}\left(\lambda_{n}+s(s-1)\right)^{-w} \\
& =\Gamma(w) \sum_{n=0}^{\infty}\left(\lambda_{n}+s(s-1)\right)^{-w}=\Gamma(w) \zeta(w, s, \Delta) \quad(\operatorname{Re}(s)>1)
\end{aligned}
$$

So we give the analytic continuation of the Mellin transformation of (2.1.D) with the present test function. For that purpose we write the trace formula in the following form: 
Lemma 3.1. The Selberg trace formula with the present test function is

$$
\operatorname{Tr}_{D}(t)=I(t)+E(t)+H(t)+C P_{1}(t)+C P_{2}(t)+C P_{3}(t),
$$

where

$$
I(t)=\frac{\operatorname{vol}(\Gamma \backslash \mathbf{H})}{4 \pi} \int_{-\infty}^{\infty} \exp \left(-t\left(r^{2}+\left(s-\frac{1}{2}\right)^{2}\right)\right) r \tanh (\pi r) d r
$$

$H(t)=\frac{1}{\sqrt{4 \pi t}} \sum_{P} \sum_{m=1}^{\infty} \frac{\log N(P)}{N(P)^{m / 2}-N(P)^{-m / 2}} \exp \left(-t\left(s-\frac{1}{2}\right)^{2}-\frac{(m \log N(P))^{2}}{4 t}\right)$,

$E(t)=\frac{1}{2} \sum_{R} \sum_{m=1}^{\nu-1} \frac{1}{\nu \sin \pi m / \nu} \int_{-\infty}^{\infty} \frac{\exp (-2 \pi r m / \nu)}{1+\exp (-2 \pi r)} \exp \left(-t\left(r^{2}+\left(s-\frac{1}{2}\right)^{2}\right)\right) d r$,

$$
\begin{gathered}
C P_{1}(t)=\left(\frac{1}{\sqrt{4 \pi t}} \log \frac{\pi^{K}}{2^{K} A}+\frac{K-K_{0}}{4}\right) \exp \left(-t\left(s-\frac{1}{2}\right)^{2}\right) \\
C P_{2}(t)=\frac{1}{\sqrt{\pi t}} \sum_{\chi} \sum_{n=1}^{\infty} \frac{\Lambda(n) \chi(n)}{n} \exp \left(-t\left(s-\frac{1}{2}\right)^{2}-\frac{(\log n)^{2}}{t}\right),
\end{gathered}
$$

$$
C P_{3}(t)=-\frac{K}{2 \pi} \int_{-\infty}^{\infty}\left(\psi\left(\frac{1}{2}+i r\right)+\psi(1+i r)\right) \exp \left(-t\left(r^{2}+\left(s-\frac{1}{2}\right)^{2}\right)\right) d r .
$$

(Here the notation CP denotes the contribution of both the continuous spectrum and the parabolic conjugacy classes.)

If $M$. denotes the Mellin transformation, it suffices to give the analytic continuation of $M \operatorname{Tr}_{D}(w)$. As $t \rightarrow \infty, \operatorname{Tr}_{D}(t)$ decays exponentially. Hence possible poles of $\zeta(w, s, \Delta)$ derive from the behavior of $\operatorname{Tr}_{D}(t)$ as $t \rightarrow 0$. So we will examine the behavior of each term of the trace formula as $t \rightarrow 0$. It is done by Kurokawa [5] when $\Gamma$ is $\operatorname{PSL}(2, \mathbf{Z})$. We will apply his method to the case $\Gamma=\Gamma_{i}(N)(i=0,1,2)$. It is obvious that $H(t)$ and $C P_{2}(t)$ decay exponentially as $t \rightarrow 0$. As for $I(t), E(t)$, and $C P_{1}(t)$, we can use the expansion of the exponential function. First $I(t)$ can be computed by integrating by parts:

$$
\begin{aligned}
I(t) & =\frac{\operatorname{vol}(\Gamma \backslash \mathbf{H})}{4 \pi} \int_{-\infty}^{\infty} \exp \left(-t\left(r^{2}+\left(s-\frac{1}{2}\right)^{2}\right)\right) r \tanh (\pi r) d r \\
& =\frac{\operatorname{vol}(\Gamma \backslash \mathbf{H})}{8 t} \int_{-\infty}^{\infty} \exp \left(-t\left(r^{2}+\left(s-\frac{1}{2}\right)^{2}\right)\right) \operatorname{sech}^{2}(\pi r) d r \\
& =\frac{\operatorname{vol}(\Gamma \backslash \mathbf{H})}{8} \sum_{n=0}^{\infty} \frac{(-1)^{n}}{n !} t^{n-1} \int_{-\infty}^{\infty}\left(r^{2}+\left(s-\frac{1}{2}\right)^{2}\right)^{n} \operatorname{sech}^{2}(\pi r) d r
\end{aligned}
$$


where the last integral converges for all $n$. So $I(t)$ has the following expansion of $t$ :

$$
I(t)=\sum_{n=-1}^{\infty} a_{n} t^{n} \quad\left(a_{n} \in \mathbf{R}\right)
$$

with $a_{-1}=\operatorname{vol}(\Gamma \backslash \mathbf{H}) / 4 \pi$.

Next $E(t)$ also can be computed as

$$
\begin{aligned}
E(t)= & \frac{1}{2} \sum_{R} \sum_{m=1}^{\nu-1} \frac{1}{\nu \sin \pi m / \nu} \int_{-\infty}^{\infty} \frac{\exp (-2 \pi r m / \nu)}{1+\exp (-2 \pi r)} \exp \left(-t\left(r^{2}+\left(s-\frac{1}{2}\right)^{2}\right)\right) d r \\
= & \frac{1}{2} \sum_{R} \sum_{m=1}^{\nu-1} \frac{1}{\nu \sin \pi m / \nu} \sum_{n=0}^{\infty} \frac{(-1)^{n}}{n !} t^{n} \\
& \quad \times \int_{-\infty}^{\infty} \frac{\exp (-2 \pi r m / \nu)}{1+\exp (-2 \pi r)}\left(r^{2}+\left(s-\frac{1}{2}\right)^{2}\right)^{n} d r,
\end{aligned}
$$

which gives the expansion of $E(t)$ as

$$
E(t)=\sum_{n=0}^{\infty} b_{n} t^{n}, \quad\left(b_{n} \in \mathbf{R}\right)
$$

Similarly, $C P_{1}(t)$ can be directly expanded as

$$
C P_{1}(t)=\sum_{n=0}^{\infty} c_{n} t^{n}+\sum_{n=0}^{\infty} d_{n} t^{n-1 / 2} \quad\left(c_{n}, d_{n} \in \mathbf{R}\right)
$$

From (3.2.I), (3.2.E), and (3.2.CP1), we have the following expansion of $\left(\operatorname{Tr}_{D}-C P_{3}\right)(t)$ as $t \rightarrow 0$ :

$$
\left(\operatorname{Tr}_{D}-C P_{3}\right)(t)=\sum_{n=-1}^{\infty} A_{n} t^{n}+\sum_{n=0}^{\infty} d_{n} t^{n-1 / 2} \quad\left(A_{n}:=a_{n}+b_{n}+c_{n}\right)
$$

Since both $\operatorname{Tr}_{D}(t)$ and $C P_{3}(t)$ decay exponentially as $t \rightarrow \infty$, possible poles of $M\left(\operatorname{Tr}_{D}-C P_{3}\right)(w)$ derive from

$$
\int_{0}^{1}\left(\sum_{n=-1}^{\infty} A_{n} t^{n+w-1}+\sum_{n=0}^{\infty} d_{n} t^{n+w-3 / 2}\right) d t=\sum_{n=-1}^{\infty} \frac{A_{n}}{n+w}+\sum_{n=0}^{\infty} \frac{d_{n}}{n+w-\frac{1}{2}}
$$

So $M\left(\operatorname{Tr}_{D}-C P_{3}\right)(w)$ has simple poles at $w=1, \frac{1}{2}, 0,-\frac{1}{2},-1,-\frac{3}{2}, \ldots$, among which the poles at nonpositive integers are those of $\Gamma(w)$. 
Next we give the analytic continuation of $M C P_{3}(w)$. Our $M C P_{3}(w)$ is

$$
\begin{aligned}
M C P_{3}(w)= & -\frac{K}{2 \pi} \int_{-\infty}^{\infty} \int_{0}^{\infty} \exp \left(-t\left(r^{2}+\left(s-\frac{1}{2}\right)^{2}\right)\right) t^{w-1} d t \\
& \times\left(\psi\left(\frac{1}{2}+i r\right)+\psi(1+i r)\right) d r \\
= & -\frac{K}{2 \pi} \Gamma(w) \int_{-\infty}^{\infty}\left(r^{2}+\left(s-\frac{1}{2}\right)^{2}\right)^{-w}\left(\psi\left(\frac{1}{2}+i r\right)+\psi(1+i r)\right) d r \\
= & -\frac{2 K}{\pi} \Gamma(w) \int_{0}^{\infty}\left(r^{2}+\left(s-\frac{1}{2}\right)^{2}\right)^{-w}(\operatorname{Re}(\psi(1+2 i r))-\log 2) d r .
\end{aligned}
$$

This integral converges in $\operatorname{Re}(w)>\frac{1}{2}$, because $\operatorname{Re}(\psi(1+2 i r))$ increases as fast as $\log r$ when $r \rightarrow \infty$. We will give the analytic continuation of this integral and examine poles of it. The last integral equals

$$
\left(s-\frac{1}{2}\right)^{1-2 w} \int_{0}^{\infty}\left(1+x^{2}\right)^{-w}(\operatorname{Re}(\psi(1+(2 s-1) x i))-\log 2) d x
$$

by $r=\left(s-\frac{1}{2}\right) x$. We devide this integral into $\int_{0}^{1}$ and $\int_{0}^{\infty}$. The former is finite for all $w \in \mathbf{C}$. The latter is equal to

$$
\frac{1}{2} \int_{0}^{1} y^{w-3 / 2}(y+1)^{-w}\left(\operatorname{Re}\left(\psi\left(1+\frac{2 s-1}{\sqrt{y}} i\right)\right)-\log 2\right) d y,
$$

where $x=y^{-1 / 2}$. The Stirling-Binet formula $[12,12.3$, p. 252] shows that

$$
\operatorname{Re}(\psi(1+i r))=\log r+\sum_{n=1}^{N} \frac{\alpha_{n}}{r^{2 n}}+R_{N}(r) \quad\left(\alpha_{n} \in \mathbf{R}\right),
$$

with $\left|R_{N}(r)\right| \leq M_{n} / r^{2 N+1}\left(r \geq \frac{1}{2}\right)$, where the $\alpha_{n}$ are constants expressed via Bernoulli numbers. Moreover, we can apply the binomial expansion formula to get

$$
(y+1)^{-w}=\sum_{k=0}^{\infty}\left(\begin{array}{c}
-w \\
k
\end{array}\right) y^{k}
$$

where

$$
\left(\begin{array}{c}
-w \\
k
\end{array}\right):= \begin{cases}\frac{-w(-w-1) \cdots(-w-k+1)}{k !} & (k \geq 1), \\
1 & (k=0) .\end{cases}
$$

Then the above integral $\int_{1}^{\infty}$ can be written as

$$
\frac{1}{2} \sum_{k=0}^{\infty}\left(\begin{array}{c}
-w \\
k
\end{array}\right) \int_{0}^{1} y^{w+k-3 / 2}\left(-\frac{\log y}{2}+\sum_{n=0}^{N} \frac{\alpha_{n}}{(2 s-1)^{2 n}} y^{n}+R_{N}\left(\frac{2 s-1}{\sqrt{y}}\right)\right) d y,
$$

where $\alpha_{0}:=\log (2 s-1)$. The integral has three terms. We will compute them in turn. The first term can be computed as

$$
-\frac{1}{2} \int_{0}^{1} y^{w+k-3 / 2} \log y d y=\frac{1}{2} \int_{0}^{1} \frac{y^{w+k-3 / 2}}{w+k-\frac{1}{2}} d y=\frac{1}{2\left(w+k-\frac{1}{2}\right)^{2}}
$$


for $\operatorname{Re}(w)>\frac{1}{2}-k$. The second term is

$$
\int_{0}^{1} y^{w+k-3 / 2} \sum_{n=0}^{N} \frac{\alpha_{n}}{(2 s-1)^{2 n}} y^{n} d y=\sum_{n=0}^{N} \frac{\alpha_{n}}{\left(w+k+n-\frac{1}{2}\right)(2 s-1)^{2 n}}
$$

for $\operatorname{Re}(w)>\frac{1}{2}-k$. The third term is holomorphic in $\operatorname{Re}(w)>-N$. Now we get the analytic continuation of the above integral, except double poles at $w=\frac{1}{2}-k(k=0,1,2, \ldots)$. So,

$$
M C P_{3}(w)=\Gamma(w)\left(\sum_{k=0}^{\infty}\left(\frac{p_{k}(w)}{\left(w+k-\frac{1}{2}\right)^{2}}+\frac{q_{k}(w)}{w+k-\frac{1}{2}}\right)+r(w)\right),
$$

where $p_{k}(w), q_{k}(w)(k=0,1,2, \ldots)$, and $r(w)$ are holomorphic functions on the whole $w$-plane. Then the following theorem is deduced.

Theorem 3.2. The spectral zeta function $\zeta(w, s, \Delta)$ has the analytic continuation to the whole $w$-plane except the following poles:

$$
\begin{aligned}
& w=1 \quad \text { order } 1 \\
& w=\frac{1}{2}-n \quad(n=0,1,2, \ldots) \quad \text { order } 2 .
\end{aligned}
$$

Remark. For a general cofinite subgroup $\Gamma$ of $G$, the only difficulty is that the scattering determinant $\varphi(s)$ is unknown. As for all the other terms in the trace formula, the above computation is effective.

\section{The DETERMinant of THE LAPLACIAN}

From the result in the previous section, the spectral zeta function $\zeta(w, s, \Delta)$ is holomorphic at $w=0$. So we define the discrete part of the determinant of the Laplacian by

$$
\operatorname{det}_{D}(\Delta+s(s-1)):=\exp \left(-\left.\frac{\partial}{\partial w}\right|_{w=0} \zeta(w, s, \Delta)\right) .
$$

Formally, it is $\prod_{n=0}^{\infty}\left(\lambda_{n}+s(s-1)\right)$. (We have assumed that $s$ is a real number larger than 1. For general complex number $s, \operatorname{det}_{D}(\Delta+s(s-1))$ will be defined in $\S 5$.) Here we take the following function as a test function of the Selberg trace formula:

$$
h\left(r^{2}+\frac{1}{4}\right):=\frac{1}{r^{2}+\frac{1}{4}+s(s-1)}-\frac{1}{r^{2}+\beta^{2}} \quad\left(\beta>\frac{1}{2}, s>1\right),
$$

which satisfies the conditions of the trace formula. The corresponding $g(u)$ is

$$
g(u)=\frac{1}{2 s-1} e^{-(s-1 / 2)|u|}-\frac{1}{2 \beta} e^{-\beta|u|} .
$$

The terms (2.1.D) and (2.4.Ci) $(i=1,2,3,4)$ in the Selberg trace formula with the present test function are denoted by $\operatorname{Tr}_{D}$ and $\operatorname{Tr}_{C i}$, respectively. Then it turns out that $\operatorname{det}_{D}(\Delta+s(s-1))$ comes from $\operatorname{Tr}_{D}(s)$. Indeed,

$$
\frac{d}{d s} \operatorname{Tr}_{D}(s)=-\sum_{n=0}^{\infty} \frac{2 s-1}{\left(\lambda_{n}+s(s-1)\right)^{2}}
$$


while

$$
\begin{aligned}
\frac{d}{d s} & \frac{1}{2 s-1} \frac{d}{d s} \log \operatorname{det}(\Delta+s(s-1)) \\
& =\frac{d}{d s} \frac{1}{2 s-1} \frac{d}{d s}\left(-\left.\frac{d}{d w}\right|_{w=0} \sum_{n=0}^{\infty}\left(\lambda_{n}+s(s-1)\right)^{-w}\right) \\
& =\left.\frac{d}{d w}\right|_{w=0} \frac{d}{d s} \frac{1}{2 s-1} \frac{d}{d s}\left(-\sum_{n=0}^{\infty}\left(\lambda_{n}+s(s-1)\right)^{-w}\right) \\
& =\left.\frac{d}{d w}\right|_{w=0} \frac{d}{d s} \sum_{n=0}^{\infty} w\left(\lambda_{n}+s(s-1)\right)^{-w-1} \\
& =\left.\frac{d}{d w}\right|_{w=0}\left(-\sum_{n=0}^{\infty} w(w+1)(2 s-1)\left(\lambda_{n}+s(s-1)\right)^{-w-2}\right) \\
& =-\sum_{n=0}^{\infty} \frac{2 s-1}{\left(\lambda_{n}+s(s-1)\right)^{2}} .
\end{aligned}
$$

Therefore,

$$
\frac{d}{d s} \operatorname{Tr}_{D}(s)=\frac{d}{d s} \frac{1}{2 s-1} \frac{d}{d s} \log \operatorname{det}_{D}(\Delta+s(s-1)) .
$$

Then the continuous part of the determinant also should satisfy

$$
\frac{d}{d s} \operatorname{Tr}_{C i}(s)=\frac{d}{d s} \frac{1}{2 s-1} \frac{d}{d s} \log \operatorname{det}_{C i}(\Delta, s) \quad(i=1,2,3,4) .
$$

First, since

$$
\operatorname{Tr}_{C 1}(s)=\left(\frac{1}{2 s-1}-\frac{1}{2 \beta}\right) \log \frac{A}{\pi^{K}}
$$

we have

$$
\frac{d}{d s} \operatorname{Tr}_{C 1}(s)=\frac{d}{d s} \frac{1}{2 s-1} \log \frac{A}{\pi^{K}}=\frac{d}{d s} \frac{1}{2 s-1} \frac{d}{d s} \log \left(\frac{A}{\pi^{K}}\right)^{s} .
$$

Hence

$$
\operatorname{det}_{C 1}(\Delta, s)=\left(\frac{A}{\pi^{K}}\right)^{s}
$$

Next,

$$
\operatorname{Tr}_{C 2}(s)=-2 \sum_{\chi} \sum_{n=1}^{\infty} \frac{\Lambda(n) \chi(n)}{n}\left(\frac{n^{-(2 s-1)}}{2 s-1}-\frac{n^{-2 \beta}}{2 \beta}\right) .
$$

Considering that the logarithmic derivative of the Dirichlet $L$-function $L(s, \chi)$ is equal to $-\sum_{n=1}^{\infty} \Lambda(n) \chi(n) / n^{s}$, we get

$$
\frac{d}{d s} \operatorname{Tr}_{C 2}(s)=\frac{d}{d s} \frac{1}{2 s-1} \frac{d}{d s} \sum_{\chi} \log L(2 s, \chi) .
$$

The desired contribution is

$$
\operatorname{det}_{C 2}(\Delta, s)=\prod_{\chi} L(2 s, \chi) .
$$


The third term of the continuous spectrum is

$$
\operatorname{Tr}_{C 3}(s)=\frac{K}{2 \pi} \int_{-\infty}^{\infty}\left\{\frac{1}{r^{2}+\left(s-\frac{1}{2}\right)^{2}}-\frac{1}{r^{2}+\beta^{2}}\right\} \psi\left(\frac{1}{2}+i r\right) d r .
$$

Our $\operatorname{det}_{C 3}(\Delta, s)$ should satisfy

$$
\frac{d}{d s} \log \operatorname{det}_{C 3}(\Delta, s)=\frac{K}{2 \pi} \int_{-\infty}^{\infty} \frac{2 s-1}{r^{2}+\left(s-\frac{1}{2}\right)^{2}} \psi\left(\frac{1}{2}+i r\right) d r .
$$

We compute this integral by comparing the one along the lower half-circle and the real axis. The integrand has only one pole at $r=-\left(s-\frac{1}{2}\right) i$ in the region, which is simple, and the residue is $i \psi(s)$. So the above integral can be computed and

$$
\frac{d}{d s} \log \operatorname{det}_{C 3}(\Delta, s)=K \psi(s)=\frac{d}{d s} \log \Gamma(s)^{K} .
$$

Therefore,

$$
\operatorname{det}_{C 3}(\Delta, s)=\Gamma(s)^{K}
$$

Similarly, as

$$
\operatorname{Tr}_{C 4}(s)=-\frac{K_{0}}{4}\left(\frac{1}{\left(s-\frac{1}{2}\right)^{2}}-\frac{1}{\beta^{2}}\right),
$$

it is easy to compute that

$$
\operatorname{det}_{C 4}(\Delta, s)=\left(s-\frac{1}{2}\right)^{-K_{0} / 2} .
$$

From (4.1.C1)-(4.1.C4) the continuous part of the determinant is

$$
\begin{aligned}
\operatorname{det}_{C}(\Delta, s) & =\prod_{i=1}^{4} \operatorname{det}_{C i}(\Delta, s)=\left(s-\frac{1}{2}\right)^{-K_{0} / 2}\left(\frac{A}{\pi^{K}}\right)^{s} \Gamma(s)^{K} \prod_{\chi} L(2 s, \chi) \\
& =\left(s-\frac{1}{2}\right)^{-K_{0} / 2} A^{s} \Gamma_{\mathbf{R}}(2 s)^{K} \prod_{\chi} L(2 s, \chi),
\end{aligned}
$$

where $\Gamma_{\mathbf{R}}(s):=\pi^{-s / 2} \Gamma(s / 2)$. The determinant of the Laplacian considering both discrete and continuous spectrum is defined by

$$
\operatorname{det}(\Delta, s):=\operatorname{det}_{D}(\Delta+s(s-1)) \operatorname{det}_{C}(\Delta, s) .
$$

\section{The complete Selberg zeta function}

In this section we take the same test function $h\left(r^{2}+\frac{1}{4}\right)$ as the previous section. We write the Selberg trace formula as

$$
\operatorname{Tr}_{D}(s)+\sum_{i=1}^{4} \operatorname{Tr}_{C i}(s)=I(s)+E(s)+H(s)+P_{1}(s)+P_{2}(s)+P_{3}(s),
$$


where the terms are corresponding to those in Theorems 2.1 and 2.3. The original Selberg zeta function (1.1) has the following property:

$$
\frac{d}{d s} H(s)=\frac{d}{d s} \frac{1}{2 s-1} \frac{d}{d s} \log Z(s) .
$$

So we try to express the derivation of each term of the trace formula as

$$
\frac{d}{d s} \frac{1}{2 s-1} \frac{d}{d s} \log X(s),
$$

with some functin $X(S)$. For $I(s), E(s)$, and $P_{i}(s)$, the corresponding $X(s)$ will be denoted by $Z_{I}(s), Z_{E}(s)$, and $Z_{P_{i}}(s)(i=1,2,3)$. First, $Z_{I}(s)$ is obtained by Vignéras [10], using the Barnes' double gamma function $\Gamma_{2}(s)$ :

$$
Z_{I}(s)=\left(\frac{\Gamma_{2}(s)^{2}(2 \pi)^{s}}{\Gamma(s)}\right)^{\mathrm{vol}(\Gamma \backslash \mathbf{H}) / 2 \pi} .
$$

Next, for $Z_{E}(s)$ we need some calculations. Our elliptic term is

$$
\begin{aligned}
E(s)=\frac{1}{2} \sum_{R} \sum_{m=1}^{\nu-1} \frac{1}{\nu \sin \pi m / \nu} \int_{-\infty}^{\infty} \frac{\exp (-2 \pi r m / \nu)}{1+\exp (-2 \pi r)} & \\
& \times\left(\frac{1}{r^{2}+\left(s-\frac{1}{2}\right)^{2}}-\frac{1}{r^{2}+\beta^{2}}\right) d r .
\end{aligned}
$$

Since

$$
\frac{d}{d s} E(s)=\frac{d}{d s} \frac{1}{2 s-1} \frac{d}{d s} \log Z_{E}(s),
$$

we get

$$
\frac{d}{d s} \log Z_{E}(s)=\frac{1}{2} \sum_{R} \sum_{m=1}^{\nu-1} \frac{1}{\nu \sin \pi m / \nu} \int_{-\infty}^{\infty} \frac{\exp (-2 \pi r m / \nu)}{1+\exp (-2 \pi r)} \frac{2 s-1}{r^{2}+\left(s-\frac{1}{2}\right)^{2}} d r .
$$

We will compute this integral by comparing with the one along the upper halfcircle and the real axis. The integrand has simple poles at $r=\left(s-\frac{1}{2}\right) i$ and $r=\left(n+\frac{1}{2}\right) i(n=0,1,2, \ldots)$, whose residues are

$$
-i \frac{\exp \left(-2 \pi\left(s-\frac{1}{2}\right) i m / \nu\right)}{1+\exp \left(-2 \pi\left(s-\frac{1}{2}\right) i\right)}
$$

at $r=\left(s-\frac{1}{2}\right) i$, and

$$
\frac{1}{2 \pi} \exp \left(-2 \pi\left(n+\frac{1}{2}\right) i \frac{m}{\nu}\right)\left(\frac{1}{s-n-1}+\frac{1}{s+n}\right)
$$


at $r=\left(n+\frac{1}{2}\right) i$. So $(5.2)$ equals

$$
\begin{aligned}
\pi i \sum_{R} \sum_{m=1}^{\nu-1} \frac{1}{\nu \sin \pi m / \nu}\left(\sum_{n=0}^{\infty} \frac{\exp \left(-2 \pi\left(n-\frac{1}{2}\right) i m / \nu\right)}{2 \pi}\right. & \left(\frac{1}{s-n-1}+\frac{1}{s+n}\right) \\
& =\sum_{R}\left(S_{1}+S_{2}\right),
\end{aligned}
$$

where we put

(5.4) $S_{1}=\sum_{m=1}^{\nu-1} \frac{i}{2 \nu \sin \pi m / \nu} \sum_{n=0}^{\infty} \exp \left(-2 \pi\left(n-\frac{1}{2}\right) i \frac{m}{\nu}\right)\left(\frac{1}{s-n-1}+\frac{1}{s+n}\right)$

and

$$
S_{2}=\sum_{m=1}^{\nu-1} \frac{\pi}{\nu \sin \pi m / \nu} \frac{\exp \left(-2 \pi\left(s-\frac{1}{2}\right) i m / \nu\right)}{1+\exp \left(-2 \pi\left(s-\frac{1}{2}\right) i\right)}
$$

By a change of variable $n \rightarrow \nu n+l(n \in \mathbf{Z}, l \in\{0,1,2, \ldots, \nu-1\}), S_{1}$ is computed as

$$
\begin{aligned}
S_{1}=\sum_{m=1}^{\nu-1} \frac{i}{2 \nu \sin \pi m / \nu} \sum_{n=0}^{\infty} \sum_{l=1}^{\nu-1} \exp \left(-2 \pi\left(l+\frac{1}{2}\right) i \frac{m}{\nu}\right) \\
\quad \times\left(\frac{1}{s+\nu n+l}+\frac{1}{s-\nu n-l-1}\right) \\
=\sum_{l=0}^{\nu-1} \sum_{m=1}^{\nu-1} \frac{i \exp \left(-2 \pi\left(l+\frac{1}{2}\right) i m / \nu\right)}{2 \nu \sin \pi m / \nu} \\
\times \sum_{n=0}^{\infty} \frac{1}{\nu}\left(\frac{1}{n+(s+l) / \nu}-\frac{1}{n+(-s+l+1) / \nu}\right) .
\end{aligned}
$$

The sum over $n$ can be expressed by the di-gamma function using the formula

$$
\psi(z)=\sum_{n=0}^{\infty}\left(\frac{1}{n+1}-\frac{1}{n+z}\right)-\gamma,
$$

where $\gamma$ is the Euler constant. Concerning the sum over $m$, we have the following lemma.

Lemma 5.1 (Fischer [2, p. 67]).

$$
\sum_{m=1}^{\nu-1} \frac{i \exp \left(-2 \pi\left(l+\frac{1}{2}\right) i m / \nu\right)}{\sin \pi m / \nu}=\nu-1-2 l \text {. }
$$


Then from (5.4), we have

$$
\begin{aligned}
\sum_{l=0}^{\nu-1} & \frac{\nu-1-2 l}{2 \nu} \frac{1}{\nu}\left(\psi\left(\frac{-s+l+1}{\nu}\right)-\psi\left(\frac{s+l}{\nu}\right)\right) \\
= & \sum_{l=0}^{\nu-1} \frac{2 l+1-\nu}{2 \nu^{2}}\left(\psi\left(\frac{s+l}{\nu}\right)-\psi\left(\frac{-s+l+1}{\nu}\right)\right) \\
= & \sum_{l=0}^{\nu-1} \frac{2 l+1-\nu}{2 \nu^{2}}\left(\psi\left(\frac{s+l}{\nu}\right)-\psi\left(\frac{s+\nu-l-1}{\nu}\right)\right. \\
& \left.+\pi \cot \left(\pi \frac{-s+l+1}{\nu}\right)\right) \\
= & \sum_{l=0}^{\nu-1} \frac{2 l+1-\nu}{2 \nu^{2}}\left(2 \psi\left(\frac{s+l}{\nu}\right)+\pi \cot \left(\pi \frac{-s+l+1}{\nu}\right)\right) .
\end{aligned}
$$

Since

$$
S_{2}=\sum_{m=1}^{\nu-1} \frac{\pi}{2 \nu \sin \pi m / \nu} \frac{\exp \left(-2 \pi\left(s-\frac{1}{2}\right) i m / \nu\right)+\exp \left(-2 \pi\left(s-\frac{1}{2}\right) i(\nu-m) / \nu\right)}{1+\exp \left(-2 \pi\left(s-\frac{1}{2}\right) i\right)}
$$

putting $a:=\exp \left(-2 \pi\left(s-\frac{1}{2}\right) i / \nu\right)$, we have

$$
\begin{aligned}
S_{2} & =\sum_{m=1}^{\nu-1} \frac{\pi}{2 \nu \sin \pi m / \nu} \frac{a^{m}+a^{\nu-m}}{1+a^{\nu}} \\
& =\sum_{m=1}^{\nu-1} \frac{\pi}{2 \nu \sin \pi m / \nu} \frac{a^{m-\nu / 2}+a^{\nu / 2-m}}{a^{\nu / 2}+a^{-\nu / 2}} \\
& =\sum_{m=1}^{\nu-1} \frac{\pi}{2 \nu \sin \pi m / \nu} \frac{\cos (2 m-1) \theta}{\cos \nu \theta},
\end{aligned}
$$

where $\theta=\pi\left(s-\frac{1}{2}\right) / \nu$. In the present situation, $\Gamma$ is a subgroup of $\operatorname{PSL}(2, \mathbf{Z})$, which has no elliptic classes other than order 2 and 3. Now we claim that (5.7) and the second term in (5.6) cancel.

Lemma 5.2. For $\nu=2,3$,

$$
\sum_{l=0}^{\nu-1} \frac{2 l+1-\nu}{\nu} \cot \left(\pi \frac{-s+l+1}{\nu}\right)=\sum_{m=1}^{\nu-1} \frac{1}{\sin \pi m / \nu} \frac{\cos (2 m-1) \theta}{\cos \nu \theta} .
$$

Proof. We need only straightforward calculationn expressing each side with $\cos \theta$ and $\sin \theta$, and using the addition theorem of trigonometric functions.

We have proved that $(5.3)$ is equal to

$$
\sum_{l=0}^{\nu-1} \frac{2 l+1-\nu}{\nu^{2}} \psi\left(\frac{s+l}{\nu}\right)
$$


Therefore

$$
Z_{E}(s)=\prod_{R} \prod_{l=0}^{\nu-1} \Gamma\left(\frac{s+l}{\nu}\right)^{(2 l+1-\nu) / \nu} .
$$

The numbers of primitive elliptic conjugacy classes in $\Gamma$ are determined by the following lemma:

Lemma 5.3 (Shimura [8, Proposition 1.43], Doi-Miyake [1, Theorem 4.2.10]). Let $a(\Gamma)$ (resp. $b(\Gamma))$ be the number of primitive elliptic conjugacy classes of order 2 (resp. 3) in $\Gamma$. Then for $\Gamma=\Gamma_{0}(N)$,

$$
\begin{aligned}
& a(\Gamma)= \begin{cases}0 & (4 \mid N), \\
\prod_{p \mid N}\left(1+\left(\frac{-1}{p}\right)\right) & (\text { otherwise }),\end{cases} \\
& b(\Gamma)= \begin{cases}0 & (9 \mid N), \\
\prod_{p \mid N}\left(1+\left(\frac{-3}{p}\right)\right) & \text { (otherwise) } .\end{cases}
\end{aligned}
$$

For $\Gamma=\Gamma_{1}(N)$,

For $\Gamma=\Gamma_{2}(N)$,

$$
a(\Gamma)=\left\{\begin{array}{ll}
0 & (N \geq 3), \\
1 & (N=1)
\end{array}, \quad b(\Gamma)= \begin{cases}0 & (N \geq 3), \\
1 & (N=1)\end{cases}\right.
$$

Note $\Gamma_{1}(2)=\Gamma_{0}(2)$.

$$
a(\Gamma)=\left\{\begin{array}{ll}
0 & (N \geq 2), \\
1 & (N=1)
\end{array}, \quad b(\Gamma)= \begin{cases}0 & (N \geq 2) \\
1 & (N=1)\end{cases}\right.
$$

Now we get the final form of $Z_{E}(s)$ :

$$
Z_{E}(s)=\left(\Gamma\left(\frac{s}{2}\right)^{-1 / 2} \Gamma\left(\frac{s+1}{2}\right)^{1 / 2}\right)^{a(\Gamma)}\left(\Gamma\left(\frac{s}{3}\right)^{-2 / 3} \Gamma\left(\frac{s+2}{3}\right)^{2 / 3}\right)^{b(\Gamma)}
$$

Remark. Lemma 5.2 is valid for any positive integer $\nu$ and $Z_{E}(s)$ is obtained for general $\nu$ by Fischer [2, Corollary 2.3.5].

For $Z_{P_{1}}(s)$ and $Z_{P_{2}}(s)$, a little calculation deduces that

$$
Z_{P_{1}}(s)=2^{-K s}
$$

and

$$
Z_{P_{2}}(s)=\left(s-\frac{1}{2}\right)^{K / 2}
$$

For $Z_{P_{3}}(s)$, we do the same as $\operatorname{det}_{C 3}$ to obtain

$$
Z_{P_{3}}(s)=\Gamma\left(s+\frac{1}{2}\right)^{-K}
$$

By (5.1) and (5.10)-(5.13), we define the complete Selberg zeta function as

$$
\widehat{Z}(s):=Z(s) Z_{I}(s) Z_{E}(s) Z_{P}(s),
$$

where $Z_{P}(s):=\prod_{i=1}^{3} Z_{P_{i}}(s)$. 
The results in the previous section and the Selberg trace formula show the main result in this paper:

Theorem 5.4. The complete Selberg zeta function has a determinant expression as

$$
\widehat{Z}(s)=e^{c+c^{\prime} s(s-1)} \operatorname{det}(\Delta, s)
$$

for some constants $c$ and $c^{\prime}$, where $\widehat{Z}(s)$ is given by the product of $(1.1),(5.1)$, and (5.10)-(5.14), while $\operatorname{det}(\Delta, s)$ is the product of (4.1.D) and (4.1. C $i)(i=$ $1,2,3,4)$.

We have supposed that $s$ is a real number greater than 1 so far. Now that the left-hand side of (5.14) has a meromorphic continuation to the whole $s$-plane, we can define $\operatorname{det}(\Delta, s)$ for a general complex number $s$, unless $\widehat{Z}(s)$ has a pole there.

It is known that zero is an eigenvalue of the Laplacian with multiplicity one. So we can write the determinant of the Laplacian as

$$
\operatorname{det}(\Delta, s)=s(s-1) \operatorname{det}^{\prime}(\Delta, s),
$$

where $\operatorname{det}^{\prime}$ means the determinant composed of nonzero eigenvalues and all the continuous spectrum. Then the following corollary is valid.

Corollary 5.5. $Z^{\prime}(1) Z_{I}(1) Z_{E}(1) Z_{P}(1)=e^{c} \operatorname{det}^{\prime}(\Delta, 1)$.

Proof. Since $Z(1)=0$, by differentiating (5.14) and substituting $s=1$, the corollary is deduced.

\section{Decision OF THE CONSTANTS}

In this section we decide the constants $c$ and $c^{\prime}$ in the determinant expression (5.14). We write the logarithm of (5.14) as

$\log Z(s)+\log Z_{I}(s)+\log Z_{E}(s)+\log Z_{C P}(s)=c+c^{\prime} s(s-1)+\log \operatorname{det}_{D}(\Delta+s(s-1))$, where

$$
\begin{aligned}
Z_{C P}(s) & :=Z_{P}(s) \operatorname{det}_{C}(\Delta, s)^{-1} . \\
& =2^{-K s}\left(s-\frac{1}{2}\right)^{\left(K_{0}+K\right) / 2} \Gamma\left(s+\frac{1}{2}\right)^{-K} \Gamma(s)^{-K}\left(\frac{\pi^{K}}{A}\right)^{s} \prod_{\chi} L(2 s, \chi)^{-1} .
\end{aligned}
$$

We examine the behavior of each side of (6.1) as $s \rightarrow \infty$. From the definition, $\log Z(s) \rightarrow 0$ as $s \rightarrow \infty$. We have the following asymptotic behavior of $\Gamma_{2}(s)$ :

$\log \Gamma_{2}(s)=-\frac{s-1}{2} \log 2 \pi+\frac{\log s}{12}-\frac{1}{12}-\zeta^{\prime}(-1)-(s-1)^{2}\left(\frac{\log s}{2}-\frac{3}{4}\right)-\frac{s}{2}+o(1)$,

which gives

$Z_{I}(s)=\frac{\operatorname{vol}(\Gamma \backslash \mathbf{H})}{2 \pi}\left(-s^{2}+s-\frac{1}{3}\right) \log s+\frac{3}{2}(s-1)^{2}+\left(\frac{1}{2} \log 2 \pi-2 \zeta^{\prime}(-1)\right)+o(1)$. 
Remark. The constant $\zeta^{\prime}(-1)$ is known by Kinkelin [4]:

$$
\begin{aligned}
\zeta^{\prime}(-1) & =\frac{1}{12}-\lim _{n \rightarrow \infty}\left(\sum_{k=1}^{n} k \log k-\left(\frac{n^{2}}{2}+\frac{n}{2}+\frac{1}{12}\right) \log n+\frac{n^{2}}{4}\right) \\
& =\frac{1}{12}-1.2824271 \cdots=0.08333 \cdots-1.2824271 \cdots=-1.19909 \cdots .
\end{aligned}
$$

From Stirling's formula, the behavior of $\log Z_{E}(s)$ is shown to be

$$
\log Z_{E}(s)=\left(\frac{a(\Gamma)}{4}+\frac{4 b(\Gamma)}{9}\right) \log s-\left(\frac{a(\Gamma)}{4} \log 2+\frac{4 b(\Gamma)}{9} \log 3\right)+o(1) .
$$

Similarly, $\log Z_{C P}(s)$ is

$\log Z_{C P}(s)=\left(\log \frac{\pi^{K}}{2^{K} A}+2 K\right) s-K \log 2 \pi-2 K s \log s+\left(\frac{2 K+K_{0}}{2}\right) \log s+o(1)$.

Next, we examine the behavior of $\log \operatorname{det}_{D}(\Delta+s(s-1))$ as $s \rightarrow \infty$. For that purpose, we have to know the behavior of $\operatorname{Tr}_{D}(t)$ as $t \rightarrow 0$. From $\S 3$,

$$
\left(\operatorname{Tr}_{D}-C P_{3}\right)(t)=\sum_{n=-1}^{\infty} A_{n} t^{n}+\sum_{n=0}^{\infty} d_{n} t^{n-1 / 2} \quad\left(A_{n}:=a_{n}+b_{n}+c_{n}\right)
$$

and

$$
M C P_{3}(w)=\Gamma(w)\left(\sum_{k=0}^{\infty}\left(\frac{p_{k}(w)}{\left(w+k-\frac{1}{2}\right)^{2}}+\frac{q_{k}(w)}{w+k-\frac{1}{2}}\right)+r(w)\right),
$$

where $a_{n}, b_{n}, c_{n}, d_{n}$ are constants, and $p_{k}(w), q_{k}(w), r(w)$ are holomorphic functions defined in $\S 3$. The inverse Mellin transformation is the following:

$$
C P_{3}(t)=\frac{1}{2 \pi i} \int_{c-i \infty}^{c+i \infty} M C P_{3}(w) t^{-w} d w \quad\left(\operatorname{Re}(c)>\frac{1}{2}\right) .
$$

The integrand has double poles at $w=\frac{1}{2}-n(n=0,1,2, \ldots)$, and simple poles at $w=-n(n=0,1,2, \ldots)$. The residue at $w=\frac{1}{2}-n$ is

$$
\lim _{w \rightarrow 1 / 2-n} \frac{d}{d w}\left(\left(w+n+\frac{1}{2}\right)^{2} M C P_{3}(w) t^{-w}\right)
$$

If we substitute (6.3), all terms but the $n$th in the infinite sum vanish by taking the limit. The residue equals

$$
\lim _{w \rightarrow 1 / 2-n} \frac{d}{d w}\left(\Gamma(w)\left(p_{n}(w)+\left(w+n-\frac{1}{2}\right) q_{n}(w)\right) t^{-w}\right)=\left(B_{n}+C_{n} \log t\right) t^{n-1 / 2},
$$

where

$$
B_{n}=\Gamma^{\prime}\left(\frac{1}{2}-n\right) p_{n}\left(\frac{1}{2}-n\right)+\Gamma\left(\frac{1}{2}-n\right)\left(p_{n}^{\prime}\left(\frac{1}{2}-n\right)+q_{n}\left(\frac{1}{2}-n\right)\right)
$$

and $C_{n}=-\Gamma\left(\frac{1}{2}-n\right) p_{n}\left(\frac{1}{2}-n\right)$. Simple poles come from $\Gamma(w)$ in (6.3). The residue at $w=-n(n=0,1,2, \ldots)$ is

$$
\lim _{w \rightarrow-n}\left((w+n) M C P_{3}(w) t^{-w}\right)=D_{n} t^{n},
$$


where

$$
D_{n}=\frac{1}{(-1)^{n} n !} \sum_{k=0}^{\infty}\left(\frac{p_{k}(-n)}{\left(-n+k-\frac{1}{2}\right)^{2}}+\frac{q_{k}(-n)}{-n+k-\frac{1}{2}}\right)+r(-n) .
$$

Comparing (6.4) with the integral along the rectangle

$$
\begin{aligned}
c-i T \rightarrow c+i T \rightarrow-(N+\varepsilon)+i T \rightarrow-(N+\varepsilon)-i T & \rightarrow c-i T \\
(T>0, \varepsilon & >0, N>0, N \in \mathbf{Z}),
\end{aligned}
$$

we obtain the expansion of $C P_{3}(t)$ :

$$
C P_{3}(t)=\sum_{n=0}^{N}\left(B_{n}+C_{n} \log t\right) t^{n-1 / 2}+\sum_{n=0}^{N} D_{n} t^{n}+O\left(t^{N}\right) \quad(t \rightarrow 0) .
$$

By (6.2) and (6.5), the behavior of $\operatorname{Tr}_{D}(t)$ as $t \rightarrow 0$ is deduced:

$$
\operatorname{Tr}_{D}(t) \approx \sum_{n=0}^{\infty}\left(B_{n}+d_{n}+C_{n} \log t\right) t^{n-1 / 2}+\sum_{n=-1}^{\infty}\left(A_{n}+D_{n}\right) t^{n},
$$

with $D_{-1}=0$. Here we put $\operatorname{Tr}_{0}(t):=\sum_{n=0}^{\infty} e^{-t \lambda_{n}}$ and

$$
\begin{aligned}
f(t):=\left(\operatorname{Tr}_{0}(t)-\left(B_{0}+d_{0}+C_{0} \log t\right) t^{-1 / 2}-\left(B_{1}+d_{1}+C_{1} \log t\right) t^{1 / 2}\right. \\
\left.-\left(A_{-1}+D_{-1}\right) t^{-1}-\left(A_{0}+D_{0}\right)\right) \times 1 / t,
\end{aligned}
$$

where all the constants are those with $s \rightarrow 1$. Then $f(t)$ is bounded near $t=0$, and it is computed that

$$
\begin{aligned}
& \log \operatorname{det}_{D}(\Delta+s(s-1))=-\left.\frac{d}{d w}\right|_{w=0} \zeta(w, s, \Delta) \\
& =-\left.\frac{d}{d w}\right|_{w=0} \frac{1}{\Gamma(w)} \int_{0}^{1} \operatorname{Tr}_{0}(t) e^{-t s(s-1)} t^{w} \frac{d t}{t}+o(1)
\end{aligned}
$$

as $s \rightarrow \infty$. We decompose $\operatorname{Tr}_{0}(t)$ into $f(t)$ and other terms, and examine the behavior of them as $s \rightarrow \infty$. Using the method of Sarnak [6], the contribution of each term to (6.7) is obtained;

$$
\begin{aligned}
& \left.\frac{d}{d w}\right|_{w=0} \frac{1}{\Gamma(w)} \int_{0}^{1}\left(B_{0}+d_{0}\right) t^{w-1 / 2} e^{-t s(s-1)} \frac{d t}{t} \\
& \quad=\left.\frac{d}{d w}\right|_{w=0} \frac{B_{0}+d_{0}}{\Gamma(w)(s(s-1))^{w-1 / 2}} \int_{0}^{s(s-1)} e^{-y} y^{w-1 / 2} \frac{d y}{y} \quad(y:=t s(s-1)) \\
& \quad=\left.\frac{d}{d w}\right|_{w=0} \frac{B_{0}+d_{0}}{\Gamma(w)(s(s-1))^{w-1 / 2}}\left(\Gamma\left(w-\frac{1}{2}\right)-\int_{s(s-1)}^{\infty} e^{-y} y^{w-1 / 2} \frac{d y}{y}\right) \\
& =\Gamma\left(-\frac{1}{2}\right)(s(s-1))^{1 / 2}\left(B_{0}+d_{0}\right)+o(1)=\Gamma\left(-\frac{1}{2}\right)\left(s-\frac{1}{2}\right)\left(B_{0}+d_{0}\right)+o(1) .
\end{aligned}
$$


Similarly,

$$
\begin{aligned}
& \left.\frac{d}{d w}\right|_{w=0} \frac{1}{\Gamma(w)} \int_{0}^{1} C_{0}(\log t) e^{-t s(s-1)} t^{w-1 / 2} \frac{d t}{t} \\
& \quad=-2 \Gamma\left(-\frac{1}{2}\right)\left(s-\frac{1}{2}\right)(\log s) C_{0}+\Gamma^{\prime}\left(-\frac{1}{2}\right)\left(s-\frac{1}{2}\right) C_{0}+o(1), \\
& \left.\frac{d}{d w}\right|_{w=0} \frac{1}{\Gamma(w)} \int_{0}^{1}\left(A_{-1}+D_{-1}\right) e^{-t s(s-1)} t^{w-1} \frac{d t}{t} \\
& =(-s(s-1)+2 s(s-1) \log s)\left(A_{-1}+D_{-1}\right)+o(1), \\
& \left.\frac{d}{d w}\right|_{w=0} \frac{1}{\Gamma(w)} \int_{0}^{1}\left(A_{0}+D_{0}\right) e^{-t s(s-1)} t^{w} \frac{d t}{t}=-2(\log s)\left(A_{0}+D_{0}\right)+o(1),
\end{aligned}
$$

and other terms tend to 0 as $s \rightarrow \infty$. Now we get the behavior of $\log \operatorname{det}_{D}(\Delta+s(s-1))$ in the right-hand side of $(6.1)$ :

$\log \operatorname{det}_{D}(\Delta+s(s-1))$

$$
\begin{aligned}
\approx & -\left(A_{-1}+D_{-1}\right) s(s-1)+2\left(A_{-1}+D_{-1}\right) s(s-1) \log s \\
& +\left(\Gamma\left(-\frac{1}{2}\right)\left(B_{0}+d_{0}\right)+\Gamma^{\prime}\left(-\frac{1}{2}\right) C_{0}\right) s-2 \Gamma\left(-\frac{1}{2}\right) C_{0} s \log s \\
& -\frac{1}{2}\left(\Gamma\left(-\frac{1}{2}\right)\left(B_{0}+d_{0}\right)+\Gamma^{\prime}\left(-\frac{1}{2}\right) C_{0}\right)-2\left(A_{0}+D_{0}-\frac{\Gamma\left(-\frac{1}{2}\right)}{2} C_{0}\right) \log s .
\end{aligned}
$$

All we have to do is compare the coefficients of both sides of the behavior of (6.1) as $s \rightarrow \infty$. By comparing the constant term,

$$
\begin{aligned}
c+ & \frac{1}{2}\left(-\Gamma\left(-\frac{1}{2}\right)\left(B_{0}+d_{0}\right)-\Gamma^{\prime}\left(-\frac{1}{2}\right) C_{0}\right) \\
\in & \frac{\operatorname{vol}(\Gamma \backslash \mathbf{H})}{2 \pi}\left(\frac{\log 2 \pi}{2}-2 \zeta^{\prime}(-1)\right)-\left(\frac{a(\Gamma)}{4} \log 2+\frac{4 b(\Gamma)}{9} \log 3\right) \\
& \quad-K \log 2 \pi+2 \pi i \mathbf{Z} .
\end{aligned}
$$

Comparing the coefficients of $s(s-1)$ shows that

$$
c^{\prime}-\left(A_{-1}+D_{-1}\right)=3 \frac{\operatorname{vol}(\Gamma \backslash \mathbf{H})}{4 \pi} \text {. }
$$

It remains to decide $B_{0}, d_{0}, C_{0}, A_{-1}$, and $D_{-1}$. We compute them in turn. From the definition,

$$
B_{0}=\Gamma^{\prime}\left(\frac{1}{2}\right) p_{0}\left(\frac{1}{2}\right)+\Gamma\left(\frac{1}{2}\right)\left(p_{0}^{\prime}\left(\frac{1}{2}\right)+q_{0}\left(\frac{1}{2}\right)\right) .
$$

The explicit form of $p_{0}(w)$ and $q_{0}(w)$ with $s \rightarrow 1$ is available from $\S 3$ :

$$
p_{0}(w)=-\frac{K}{2 \pi}\left(\frac{1}{2}\right)^{1-2 w} \text { and } q_{0}(w)=0 .
$$

Hence

$$
p_{0}\left(\frac{1}{2}\right)=\frac{K}{2 \pi}, \quad p_{0}^{\prime}\left(\frac{1}{2}\right)=-\frac{K \log 2}{\pi}, \quad \text { and } \quad q_{0}\left(\frac{1}{2}\right)=0
$$


As the constant $d_{0}$ is the coefficient of $t^{-1 / 2}$ in (3.1.CP 1$)$,

It is easy to see that

$$
d_{0}=\frac{1}{2 \sqrt{\pi}} \log \frac{\pi^{K}}{2^{K} A} .
$$

$$
C_{0}=-\Gamma\left(\frac{1}{2}\right) p_{0}\left(\frac{1}{2}\right)=\frac{K \Gamma\left(\frac{1}{2}\right)}{2 \pi}, \quad A_{-1}=a_{-1}=\frac{\operatorname{vol}(\Gamma \backslash \mathbf{H})}{4 \pi},
$$

and $D_{-1}=0$. Now we have the conclusion.

Proposition 6.1. The constants appearing in the determinant expression (5.14) are as follows:

$$
\begin{aligned}
c \in & \frac{\operatorname{vol}(\Gamma \backslash \mathbf{H})}{2 \pi}\left(\frac{\log 2 \pi}{2}-2 \zeta^{\prime}(-1)\right) \\
& -\left(\frac{a(\Gamma)}{4} \log 2+\frac{4 b(\Gamma)}{9} \log 3\right)+K \log \frac{2}{\sqrt{\pi} e}+\sqrt{\pi} \log \frac{\pi^{K}}{2^{K} A}+2 \pi i \mathbf{Z},
\end{aligned}
$$

and $c^{\prime}=\operatorname{vol}(\Gamma \backslash \mathbf{H}) / \pi$.

\section{REFERENCES}

1. K. Doi and T. Miyake, Automorphic forms and number theory, Kinokuni-ya, 1976. (Japanese)

2. J. Fischer, An approach to the Selberg trace formula via the Selberg zeta function, Lecture Notes in Math., vol. 1253, Springer, 1987.

3. M. N. Huxley, Scattering matrices for congruence subgroups, in Modular Forms (R. A. Rankin, ed.), Ellis Horwood, 1984.

4. $\mathrm{H}$. von Kinkelin, Ueber eine mit der Gammafunktion verwandte Transcendente und deren Anwendung auf die Integralrechnung, J. Reine Angew. Math. 57 (1860), 122-138.

5. N. Kurokawa, Parabolic components of zeta functions, Proc. Japan Acad. Ser. A Math. Sci. 64 (1988), 21-24.

6. P. Sarnak, Determinants of Laplacians, Comm. Math. Phys. 110 (1987), 113-120.

7. A. Selberg, Harmonic analysis and discontinuous groups on weakly symmetric Riemannian surfaces with applications to Dirichlet series, J. Indian Math. Soc. 20 (1956), 47-87.

8. G. Shimura, Introduction to the arithmetic theory of automorphic functions, Princeton Univ. Press, Princeton, N.J., 1971.

9. A. B. Venkov, Spectral theory of automorphic functions, Proc. Steklov Inst. Math. 153 (1982), 1-163.

10. M.-F. Vignéras, L'équation fonctionnelle de la fonction zêta de Selberg du groupe modulaire $\operatorname{PSL}(2, \mathbf{Z})$, Astérisque 61 (1979), 235-249.

11. A. Voros, Spectral functions, special functions, and the Selberg zeta function, Comm. Math. Phys. 110 (1987), 439-465.

12. E. T. Whittaker and G. N. Watson, A course of modern analysis, Cambridge Univ. Press, 1927.

13. S. Koyama, Determinant expression of Selberg zeta functions. II, Trans. Amer. Math. Soc. (to appear).

Department of Mathematics, Tokyo Institute of Technology, Oh-OKayama, Meguro$\mathrm{KU}$, TOKYO, 152, JAPAN

Current address: Department of Mathematics, Faculty of Science and Technology, Keio University, 14-1, Hiyoshi, 3 chome, Kohoku-ku, Yokohama 223, Japan 